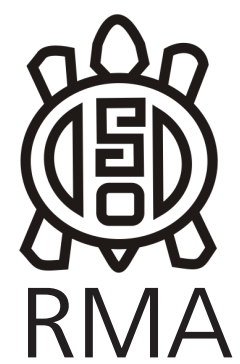

Arqueología

\title{
Zooarqueología del sitio formativo Soria 2, valle de Yocavil (Catamarca), siglo I d.C.
}

\author{
Carlos R. Belotti López de Medina
}

CONICET, Museo Etnográfico "Juan B. Ambrosetti", Facultad de Filosofía y Letras, Universidad de Buenos Aires. E-mail: crbelotti@yahoo.com.ar

\begin{abstract}
Resumen
En este trabajo presentamos un análisis exhaustivo de las arqueofaunas recuperadas en el sitio Soria 2 (sudeste del valle de Yocavil o Santa María, provincia de (atamarca), durante los trabajos de campo realizados entre los años 2002 y 2006. El sitio es una estructura formada por al menos dos recintos y fue identificado como un espacio doméstico. Los conjuntos estudiados provienen de un depósito arqueológico con abundante contenido cultural y datado en 1940 años ARCP, lo que ubica a este contexto en los inicios del Período Formativo del valle. Los principales recursos animales fueron los camélidos silvestres (Lama guanicoe y Vicugna vicugna) y domésticos (Lama glama), con un predominio marcado de ejemplares subadultos de acuerdo a las estimaciones de número mínimo de individuos (MNI). Los resultados presentados contribuirán a comprender la diversidad y evolución en la explotación de recursos faunísticos durante los primeros siglos de la economía agropastoril en el área.
\end{abstract}

Palabras clave: Yocavil; Formativo; Zooarqueología; Soria 2.

Zooarchaeology of the Formative site Soria 2, Yocavil Valley (Catamarca).First century A.D.

\begin{abstract}
We present here an analysis of the faunal remains recovered at the Soria 2 site (Yocavil valley, Catamarca), along the fieldwork seasons from 2002 to 2006. The site is an architectural structure composed by at least two rooms and was identified as a household. The assemblages reviewed here were found at an archaeological deposit with abundant cultural remains and a radiocarbon date of 1940 RCYBP, situating this context at the very beginnings of the Formative Period of Yocavil. The most important faunal resource were domestic and wild Camelidae species (Lama glama, Lama guanicoe and Vicugna vicugna), with a marked preference for young animals according to the estimations of the Minimal Number of Individuals. These results will help to understand the diversity and evolution of the exploitation of faunal resources at the first centuries of the agropastoralist economy in the area.
\end{abstract}

Key words: Yocavil; Formative; Zooarchaeology; Soria 2.

Una de las principales aspiraciones de la arqueología es la reconstrucción de los sistemas sociales prehistóricos. En nuestro caso el enfoque adoptado para abordar semejante tarea se nutre de diversas corrientes del materialismo histórico, en especial de los aportes de la arqueología social latinoamericana (Bate 1998), del posibilismo defendido por Bruce Trigger (1991) y del análisis del poder aportado por Eric Wolf (1990). Vemos así a la praxis como la fuerza constitutiva y transformadora de la sociedad, aunque su ejercicio esté sujeto a restricciones objetivas y subjetivas (Trigger 1991).

En el largo plazo parece evidente que los mayores condicionantes a la acción humana se derivan de la base económica, de la unidad dialéctica entre relaciones de producción y magnitud de las fuerzas productivas. Un claro ejemplo es la reproducción de la fuerza laboral incluida la subsistencia-, en tanto limita el surgimiento de las clases sociales por dos vías diferentes. Una porque el tiempo socialmente requerido para su realización restringe la generación de plusproducto enajenable. Dos, porque ciertas formas de explotación del trabajo son más adecuadas que otras, según si los trabajadores controlan o no los medios de producción necesarios para su subsistencia. Con estas consideraciones en mente, tratamos de abordar en trabajos anteriores el problema de la reproducción material de las primeras comunidades agroalfareras del valle de Santa María o Yocavil (Provincia de Catamarca, Argentina) y su posterior evolución (Belotti 2007, 2010a). En especial nos interesamos por los cambios ocurridos en la subsistencia, vistos a través de las tendencias diacrónicas en el registro zooarqueológico. 
En este trabajo presentamos los resultados del estudio de las arqueofaunas recuperadas en el sitio Soria 2, una unidad residencial ubicada en el sector sudeste del valle de Yocavil. Las dataciones obtenidas hasta el momento lo sitúan como uno de los sitios agropastoriles más tempranos del valle, en los inicios mismos del Período Formativo (ca. siglo I D.C.). En la última década la zooarqueología de Yocavil ha crecido de manera considerable. Entre los aportes más importantes están los trabajos en los sitios formativos Bañado Viejo y Morro de las Espinillas (Izeta 2007; Izeta y Scattolin 2001), y en los sitios tardíos Rincón Chico 15 y Las Mojarras 1 (Pratolongo 2008). Los resultados que damos a conocer aquí contribuirán al entendimiento del modo de vida de los habitantes de Yocavil en los comienzos de la economía agropastoril, así como a la formación de una base de datos adecuada para detectar los cambios ocurridos en la subsistencia entre finales del último milenio A.C. y la conquista incaica (ca. mediados del siglo XV D.C.).

\section{Antecedentes}

\section{El Formativo Surandino}

Nuestra investigación se sitúa en los albores del Período o estadio Formativo del valle de Yocavil. En su acepción tradicional el Formativo viene a ser un equivalente americano del Neolítico, y como aquel involucraría niveles característicos de desarrollo técnico, económico y sociopolítico, que fueron en parte posibilitados por la difusión de cultígenos y otras innovaciones (González 1998). Para los Andes meridionales la primera definición de esta etapa la debemos a González y Pérez (González 1998; González y Pérez 1966). Posteriormente el Formativo del NOA sería redefinido sobre la base de criterios diferentes por Núñez Regueiro (1974), Raffino (1990) y Olivera (2001), entre otros. Aunque estas construcciones acusan preocupaciones teóricas distintas, coinciden grosso modo en cuanto a los indicadores arqueológicos y el contenido sociocultural del Formativo: una subsistencia mixta que combinaba la caza y recolección con la producción de alimentos (agricultura y pastoreo), sedentarismo y establecimiento de aldeas permanentes y difusión de innovaciones tecnológicas como la alfarería, la fundición de metales y el telar (e. g. Albeck 2000; Olivera 2001; Tarragó 1992, 1999).

Desde una perspectiva evolucionista el Formativo representa un momento de síntesis, o se si quiere una revolución, en la que cobra forma un tipo de sociedad cualitativamente diferente a la de los cazadoresrecolectores del Holoceno. Sin embargo nos encontramos en la necesidad de admitir que las sociedades o etapas Formativas del continente no conforman una clase natural. Este tipo de esquemas evolucionistas no representan un encadenamiento de desarrollos históricos inevitables, sino que a nuestro entender la existencia de etapas homotaxiales resulta de una convergencia evolutiva por el interjuego de restricciones internas y externas análogas (Trigger 1991), sumada a la difusión o transferencia lateral de innovaciones culturales.

Siguiendo la propuesta original de Núñez Regueiro (1974), tratamos de definir el Formativo por los cambios ocurridos en el modo de producción. A modo de hipótesis, consideramos al Formativo Surandino y al Período Temprano del NOA como un caso particular del desarrollo inicial de la Formación Económico Social (FES) Tribal (Belotti 2007, 2010a) ${ }^{1}$. Según el modelo elaborado por Vargas (1985, 1987), Sarmiento (1986) y Bate (1998), las sociedades tribales se caracterizan por una técnica agropecuaria o de almacenamiento desarrollada, la propiedad comunal de la tierra y una organización doméstica del trabajo y el consumo. Las principales contradicciones de su modo de producción radican en el crecimiento demográfico, la necesidad de un plusproducto agrícola y la conformación de sistemas regionales de intercambio (Sarmiento 1986; Vargas 1985, 1987), a las cuales deberíamos sumar el control sobre la capacidad reproductiva de las mujeres, sobre su fuerza laboral y la de sus descendientes, así como la explotación estratégica de las relaciones de parentesco para crear diferencias de poder y riqueza (Meillasoux 1977; Wolf 1987). Según las circunstancias ambientales e históricas, el desarrollo de estas contradicciones puede resolverse en la expansión de las aldeas por fisión, o en la intensificación económica y el eventual surgimiento de cacicazgos o jefaturas (chiefdoms) (Vargas 1985). Esta segunda trayectoria caracterizaría a un estadio superior de la FES Tribal, que puede derivar a su vez en el surgimiento de las primeras sociedades clasistas y estatales (Bate 1998).

En el NOA el Formativo comienza entre el 1000 y el 600 A.C., con la aparición de los primeros asentamientos agroalfareros. La culminación y las subdivisiones de este Período varían entre los distintos investigadores que trataron el asunto. En la formulación original de González y Pérez (1966) el Formativo surandino concluía entre los años 700 y 800 de la Era. Por su parte Núñez Regueiro limitó la extensión de lo que él denominaba Formativo Inferior hasta el siglo VI D.C., cuando lo sucedería un Período Formativo Medio o de Integración Regional, que llega hasta mediados del siglo IX D.C. (Núñez Regueiro 1974; Tartusi y Núñez Regueiro 1993). En sus trabajos más recientes, Tartusi y Núñez Regueiro abandonaron el concepto de Integración Regional (Núñez Regueiro y Tartusi 2001; Tartusi y Núñez Regueiro 2002), y volvieron a utilizar denominaciones como Formativo Medio o Superior (v. Núñez Regueiro 1974), que consideraron equivalentes al Período Medio de Bennett (1948) y de González (González 1979, 1998). En ambos casos el principal jalón de esta nueva fase o Período es la cultura

\footnotetext{
1 Bajo el concepto de FES Tribal incluimos también las formulaciones de Meillasoux (1977) en torno al modo de producción doméstico y de Wolf sobre el modo de producción basado en el parentesco (1987).
} 
de la Aguada, como manifestación material de un culto religioso cuya esfera de influencia abarcó distintos valles del NOA. En la secuencia propuesta por Raffino (1990) el Formativo se extiende entre el 500 A.C. y el 900 D.C., y se divide en dos sub-períodos: el Formativo Inferior (500 A.C. a 400 D.C.) y el Superior (400 a 900 D.C.). De acuerdo a Scattolin, ninguna de estas subdivisiones del Formativo sería adecuada para nuestra área de estudio, dada la escasez de evidencias que nos remitan al fenómeno Aguada en Yocavil (Scattolin 2000, 2006, 2007; Tarragó y Scattolin 1999). Por esta razón propuso utilizar el Formativo en un sentido amplio, y extenderlo hasta fines del primer milenio de la Era (Scattolin 2007). En todas las propuestas, alrededor del siglo X D.C. comenzaría el Período Tardío o de Desarrollos Regionales, seguido luego por los Períodos Inka (ca. mediados del siglo XV D.C.) e Hispano-Indígena (ca. siglo XVI).

En un trabajo reciente, Scattolin sintetizó las principales tendencias del Formativo, con énfasis en el sur de los valles Calchaquíes -faldeos occidentales del Aconquija y los valles de Yocavil y del Cajón- (Scattolin 2006), área para la cual formuló además una secuencia cerámica de tres fases (Scattolin 2007). Entre los siglos X A.C. y V D.C. se establecieron las primeras aldeas en los lugares más adecuados para la agricultura o el pastoreo, y su expansión en el área valliserrana ocurriría por fisión (Scattolin 2006). Desde un primer momento se observan distintas modalidades de ocupación del paisaje: las plantas de los recintos pueden ser ortogonales $u$ ovales, las viviendas pueden estar agrupadas o dispersas entre los campos de cultivo, presencia o ausencia de construcciones públicas de naturaleza cultual, etcétera. Esto podría indicar la confluencia de diferentes tradiciones y etnicidades en la primera colonización aldeana, así como la objetivación de principios estructurales diferentes (Scattolin 2006). Al menos desde el siglo $V$ D.C. se haría evidente una intensificación de las actividades agropecuarias, visible por ejemplo en la limpieza y despedregado de grandes terrenos o en la construcción de sistemas de riego (Scattolin 2006).

A diferencia de lo que ocurre en Tafí, Campo del Pucará o Ambato, no hay evidencias en el sur de los valles Calchaquíes de arquitectura pública monumental o de centros ceremoniales durante el primer milenio. Alrededor del siglo IX D.C. surge en Santa María un nuevo tipo de ordenación territorial, en sitios como Morro de las Espinillas y Morro del Fraile: poblados aglomerados, localizados sobre mesetas y cercados por una valla que restringe el acceso. Scattolin $(2001,2006)$ propone que esta diferencia respondería a una modalidad distinta de acumulación de capital simbólico y diferenciación social, que se manifiesta en la edificación como medio de objetivación y reproducción: fijación de las relaciones sociales y mayor control sobre la fuerza de trabajo (Scattolin 2001, 2006). En el milenio siguiente, durante los Desarrollos Regionales, esta ordenación alcanzaría nuevas cotas de complejidad con los asentamientos fortificados al estilo de Quilmes o Rincón Chico. Para este Período se ha propuesto la existencia de sociedades jerarquizadas en el valle de Yocavil y en otras regiones del NOA (e. $g$. Tarragó 2000), comparables a cacicazgos o jefaturas.

Para finalizar, quisiéramos advertir que el desarrollo de la FES tribal no es un proceso lineal y armónico, y lo mismo podría decirse de la mayoría de las periodizaciones y los modelos procesuales de otros autores citados aquí. Para hacer inteligible un fenómeno cualquiera, la ciencia debe abstraer aquellas regularidades que considera relevantes en función de una teoría. Los esquemas evolucionistas destacan el surgimiento y proliferación de sistemas sociales cada vez más complejos a lo largo de la flecha del tiempo, al precio de establecer cortes arbitrarios sobre el continuo de la historia. Su aplicación al registro arqueológico acarrea dos riesgos: que se considere a los Períodos como bloques homogéneos entre los que existe algún quiebre ontológico, y que se confunda a la secuencia con un progreso ininterrumpido. Sobre el primero, téngase presente que las trayectorias de las sociedades humanas son muchas, y en cada momento pueden coexistir e interactuar sociedades con niveles diferentes de complejidad (e. g. Wolf 1987). Respecto al progreso, todos los sistemas decaen en el tiempo y las formas de organización social más complejas pueden estancarse o desaparecer.

\section{El sitio Soria 2}

El valle de Santa María o Yocavil (Catamarca) se extiende unos 100 Kilómetros en sentido $\mathrm{N}$-S, y se ubica en el extremo nororiental de la provincia de Catamarca (Departamento de Santa María) y el ángulo noroeste de la provincia de Tucumán (Departamento de Tafí). Está limitado hacia el oeste por las sierras del Cajón y al este por las sierras del Aconquija. En su extremo meridional se une con el valle de Cajón. Los conjuntos analizados en este trabajo provienen del sitio Soria 2, una unidad residencial que se asienta sobre la meseta de Andalhuala, al pie de las sierras del Aconquija y en el sector sudeste del valle (Figuras 1 y 2) (Álvarez Larrain et al. 2009; Palamarczuk et al. 2007).

La unidad residencial está conformada por al menos dos recintos ortogonales adosados, alineados en dirección este-oeste, y de cuyos muros solo se conservan unas líneas de piedra que apenas afloran sobre la superficie del terreno (Palamarczuk et al. 2007) (Figura 3). Estos fueron denominados Recintos 1 y 2, respectivamente (R1 y $R 2$ en forma abreviada).

Recinto 1: mide unos $8 \times 8$ metros y está delimitado por muros dobles al norte y al este, y por un muro simple hacia al sur. El sector donde debería hallarse el muro oeste no ha sido excavado aún. Las excavaciones se limitaron a la mitad sur de la habitación y cubren una superficie cercana 


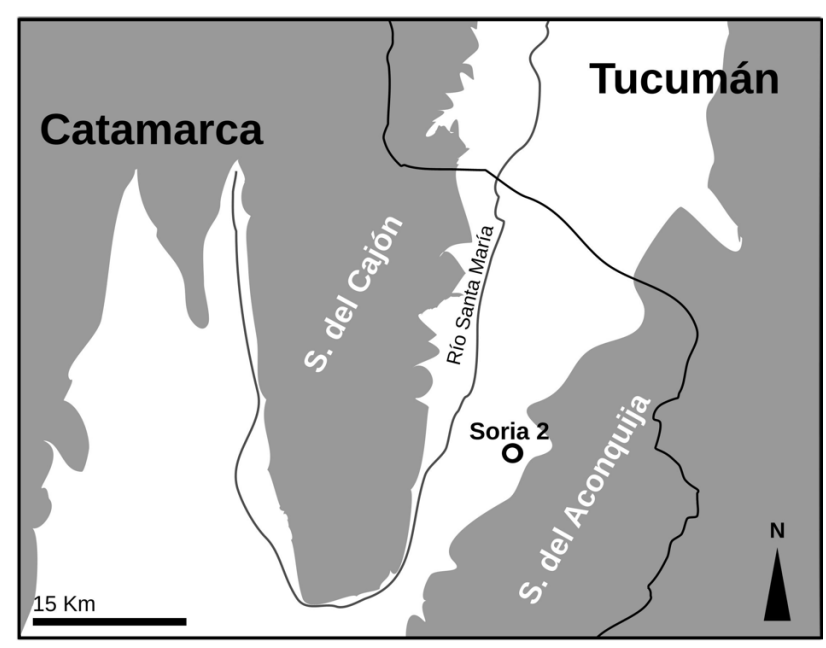

Figura 1. Localización del sitio Soria 2 en el valle de Santa María.

Figure 1. Location of Soria 2 site in the Santa María Valley

a los $32 \mathrm{~m}^{2}$. A una profundidad de entre 20 y $40 \mathrm{~cm}$ se detectó un depósito arqueológico con abundante material cultural, el cual tiene una potencia media de $30 \mathrm{~cm}$ (Weber Sanguinetti 2006). Este contenía además varias estructuras y rasgos, como agujeros de poste, un pozo de residuos y un fogón plano de $90 \times 65 \mathrm{~cm}$ (Figura 3). La presencia de estas y otras evidencias, como los artefactos cerámicos rotos in situ y depositados horizontalmente, nos llevaron a interpretar este depósito como un piso de ocupación. Del fogón se obtuvo una muestra de carbón que fue datada en $1940 \pm 80$ años ARCP (LP-1541). La fecha calibrada con dos sigmas utilizando el software CALIB 2.0 es 103 cal A.C. a cal 310 D.C., lo que haría de Soria 2 el contexto doméstico más antiguo excavado en el valle de Yocavil hasta el momento. Del pozo de residuos se obtuvo una segunda muestra para datación, unas ramas carbonizadas, con un resultado de $3460 \pm 70$ años ARCP (LP-1801). Este fechado es problemático, aunque es posible que el depósito formativo se asiente sobre ocupaciones más antiguas y que el material fechado haya sido removido por la actividad humana (Spano 2008). Al

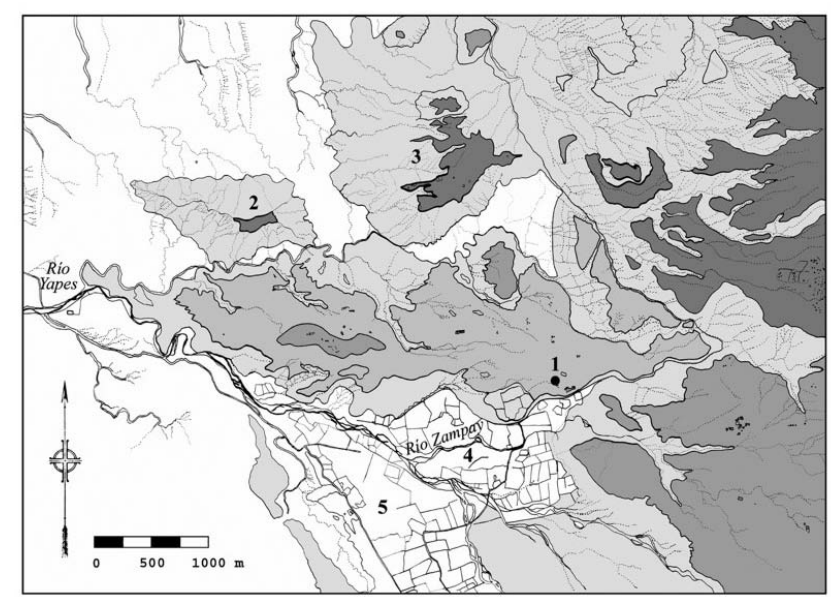

Figura 2. Meseta de Andalhuala

Figura 2. Andalhuala Plateau presente está prevista la realización de nuevas dataciones, con el objeto de refinar la cronología de Soria 2.

Recinto 2 (R2): tiene unos 6 metros de largo y todos los muros son dobles. Se excavó una cuadrícula de 2 $\times 2$ metros en su esquina sudoeste, en la cual pudo identificarse un piso de ocupación a una profundidad de entre 35 y $45 \mathrm{~cm}$, con una potencia de 15 a $30 \mathrm{~cm}$. La cerámica que contenía era similar a la del piso del Recinto 1 , lo que nos permite proponer a modo de hipótesis que ambas unidades estratigráficas son contemporáneas. En los años 2009 y 2010 se ampliaron las excavaciones hasta cubrir la mayoría del R2. Los materiales recuperados están bajo análisis.

En algún momento posterior a su uso residencial, ambos recintos se utilizaron con fines ceremoniales (Palamarczuk et al. 2007). En el R1 se encontraron dos inhumaciones, que denominamos entierros 1 y 3 (Figura 3). El entierro 1 fue hallado en un pequeño compartimiento, formado por una línea de piedras que cerraba a la esquina sudeste del recinto. Consistía en los restos desordenados de un niño, parcialmente cubiertos por la mitad de una olla ordinaria. Una parte de los huesos estaba contenida en un puco gris pulido. El entierro 3 tuvo lugar en el sector sudoeste del R1 y pertenecía a un neonato, cuyos restos fueron depositados en una olla ordinaria. El entierro 2 era similar a este último, y fue encontrado en la esquina sudoeste del R2 (Palamarczuk et al. 2007).

En la sección excavada del piso de R1 se recuperaron 8707 fragmentos de cerámica, que fueron analizados por Baigorria Di Scala y Spano (Baigorria Di Scala 2009; Baigorria Di Scala y Spano 2007; Spano 2008). Los tiestos fueron discriminados en dos conjuntos principales. 1) La cerámica ordinaria, con pastas de compactación media a baja, cocidas en atmósfera oxidante y con paredes de un grosor medio entre 8 y $12 \mathrm{~mm}$. Esta incluye formas cerradas (e.g. ollas) y abiertas (e.g. pucos). 2) La cerámica fina, de pasta compacta y en su mayoría de cocción reductora y color pardo-grisáceas-negruzcas y negras, aunque algunas piezas fueron cocidas en atmósfera oxidante y son de color ante y marrón. La forma predominante son los pucos, aunque también se identificaron fragmentos de vasos, pipas y botellones (Baigorria Di Scala 2009; Baigorria Di Scala y Spano 2007; Spano 2008).

El conjunto lítico recuperado en los sectores excavados de los pisos de ocupación de ambos recintos asciende a 996 artefactos, entre los cuales hay 20 núcleos, ocho artefactos formatizados, nueve artefactos no formatizados y el resto son desechos de talla (Carbonelli 2009). La materia prima más importante es la andesita (82\% del conjunto), seguida en proporciones mucho menores por rocas metamórficas, cuarzo y obsidiana (Carbonelli 2009). En este trabajo presentamos el estudio de los materiales faunísticos recuperados hasta enero 


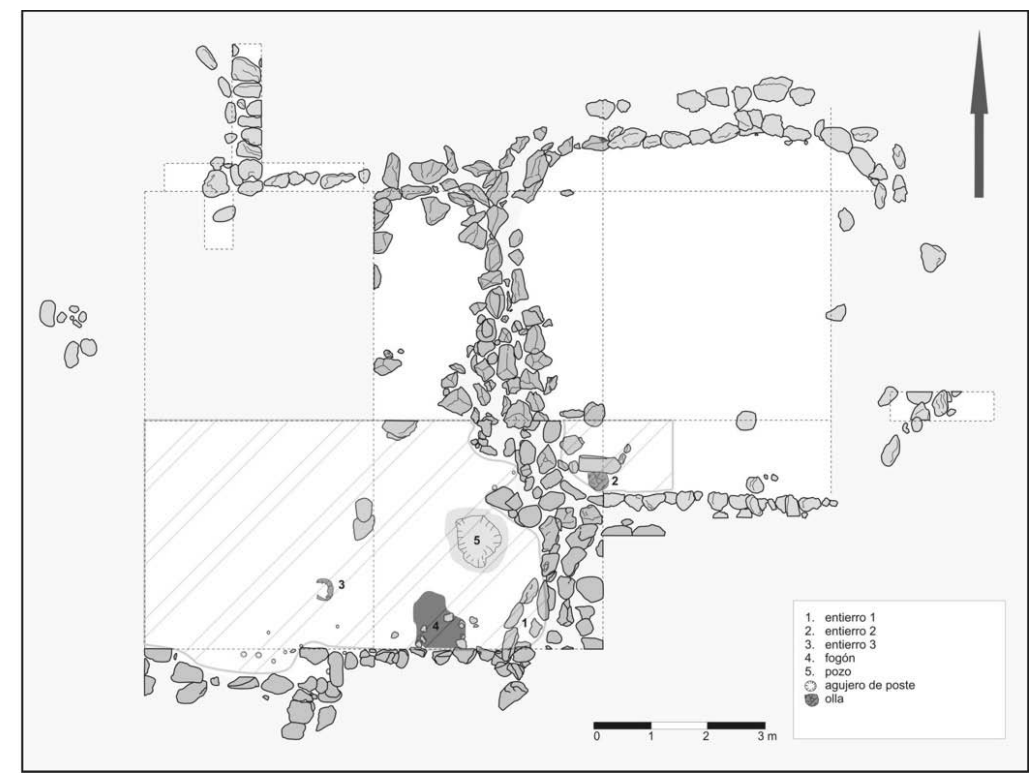

Figura 3. Planta y área excavada de Soria 2.

Figure 3. Map showing excavated area in Soria 2.

del 2006 en los depósitos formativos o pisos de ambos recintos.

\section{Metodología}

La alimentación es la culminación de una cadena de actividades organizadas de acuerdo a las relaciones sociales de producción y la división del trabajo, y cuya principal función es la reproducción de la fuerza laboral humana. La arqueozoología permite por lo tanto abordar aspectos de la base material como los medios de producción, los procesos de trabajo o la distribución de satisfactores básicos. Siguiendo a Lyman (1994) adoptamos aquí un enfoque tafonómico, entendiendo por tal que uno de los primeros objetivos de la investigación es determinar los diferentes agentes que estructuraron el conjunto antes y después de su ingreso a la litosfera, incluyendo las propias actividades de los seres humanos. En este sentido, consideramos a la tafonomía como un caso especial de la "historia de los contextos arqueológicos" (sensu Bate 1998).

Aunque los niveles de relleno contenían algún material faúnístico, el cual también fue identificado, aquí nos limitaremos a presentar los resultados obtenidos para los depósitos formativos -o pisos- de los Recintos 1 y 2. Para la identificación de los especímenes óseos se utilizaron la colección osteológica de referencia del Instituto de Arqueología (Facultad de Filosofía y Letras, Universidad de Buenos Aires) y diversos atlas osteológicos de animales domésticos y silvestres (e. g. Benavente et al. 1993; Pacheco Torres et al. 1979). Se consideraron identificables todos los huesos o fragmentos que como mínimo pudieran asignarse a alguna región anatómica, y a nivel taxonómico solo aquellos especímenes que se caracterizaron al nivel de orden o inferior.

En el caso de los materiales identificados como Camelidae se aplicó además un análisis morfométrico multivariado, con el objetivo de diferenciar animales silvestres y domésticos. Se utilizaron las guías de mediciones de Kent (1982), Elkin (1996) e Izeta (2007). Las medidas obtenidas se analizaron luego siguiendo los criterios aplicados anteriormente por Menegaz et al. (1988) e Izeta (2007): 1) se obtuvo para cada elemento una matriz de similitud con el coeficiente de distancia Manhattan o City-Block y; 2) se implementaron sobre estas matrices las técnicas de análisis de conglomerados (UPGMA) y de componentes principales. Se utilizó el software Statistica v6.0 (Statsoft 2004).

Los restos fueron asignados además a diferentes categorías de tamaño corporal, según la clasificación propuesta por Izeta (2007) para el Noroeste argentino. Esta escala comprende cinco clases de tamaño y peso para vertebrados: Clase 1, animales muy pequeños ( $e$. g. Ctenomys sp.). Clase 2, animales pequeños (e. g. Lagidium viscacia y Chaetoprhractus vellerosus). Clase 3, animales medianos (e. g. Pterocnemia pennata). Clase 4, vertebrados grandes (e. g. Hippocamelus antisensis, Vicugna vicugna y Lama sp.). Clase 9, tamaño indeterminado. También se registraron caracteres diagnósticos de la edad de muerte de los animales, como fusión de las epífisis, erupción y desgaste dentario y sutura de los huesos del cráneo (e. g. Kent 1982; Wheeler 1982).

Se identificaron las alteraciones culturales y naturales que afectaron a los restos óseos, como improntas de carnívoros, roedores y raíces, marcas de procesamiento, morfología de las fracturas, modificaciones del color y superficie de los huesos por termoalteración, etcétera. Se siguieron los criterios derivados de diversos estudios actualísticos, y sintetizados por Lyman (1994) y Mengoni (1999). En el caso de los especímenes asignados a las clases de tamaño corporal 3 y 4, se registró grado de meteorización de acuerdo a los estadios propuestos por 
Behrensmeyer (1978).

Para la cuantificación de las observaciones se utilizaron las siguientes unidades: número de especímenes (NSP); número de especímenes identificados (NISP); número mínimo de elementos (MNE), calculado por los métodos de suma de fracciones y de zonas diagnósticas, tomando para cada caso el valor más alto obtenido; número mínimo de individuos (MNI); y mínimo de unidades anatómicas (MAU y MAU\%) (Binford 1978, 1981; Izeta 2007; Mengoni 1999).

\section{Resultados}

El conjunto recuperado en el Recinto 1 está conformado por 3877 especímenes. En la tabla 1 se resumen los resultados de su identificación, con 766 especímenes caracterizados taxonómicamente (19,8\%) y otros 669 asignados a alguna clase de tamaño corporal. La mayoría de los restos identificados pertenecen a Camelidae, Artiodactyla y vertebrados grandes indeterminados (clases 3-4 y 4). Se registró el grado de meteorización de los especímenes pertenecientes a vertebrados con un peso superior a $5 \mathrm{Kg}$ (i. e. clases 3 a 4), siguiendo los lineamientos establecidos por Behrensmeyer (1978). Solo en el 9,73 \% de los casos el hueso o fragmento considerado se encontraba en un estadio superior a 0 (NSP 132, de un total de 1357). Tampoco tuvieron demasiada incidencia otros agentes naturales, ya que solo 18 especímenes presentaron marcas de roedores, 13 de carnívoros y 62 de raíces. Como veremos más adelante, tampoco hay evidencias de procesos destructivos mediados por densidad ósea (e. g. acción carnívoros).

Dada la menor extensión de las excavaciones, el conjunto recuperado en el Recinto 2 asciende solo a 171 especímenes (Tabla 2) de los cuales pudieron identificarse 69. Nuevamente se nota un predominio de los ungulados (Camelidae y Artiodactyla). De los restos asignados a las clases 3 y 4, la mayoría no presenta indicios de meteorización (NSP 101).

En lo que sigue presentaremos un análisis más detallado del subconjunto formado por los restos de Camelidae del Recinto 1.

\section{Familia Camelidae (Recinto 1)}

Está conformado por 404 especímenes, y el número mínimo de individuos ( $\mathrm{MNI}$ ) estimado es nueve: seis animales subadultos y tres animales adultos. El MNI de máxima distinción para animales adultos, obtenido en este caso a partir de la identificación de tipos morfológicos específicos, asciende a cuatro y está conformado de la siguiente manera: un individuo de Lama glama, dos de Lama guanicoe y uno de Vicugna vicugna.

En la Tabla 3 y la Figura 4 se presenta la frecuencia de partes esqueletarias, calculada según distintas unidades. Para su análisis e interpretación nos basaremos en la cuantificación del mínimo número de unidades anatómicas (MAU y MAU\%). En apariencia los elementos apendiculares predominan sobre los axiales, situación que difiere a la registrada en puestos y viviendas de pastores modernos de la Puna, donde lo común es una mayor representación del esqueleto axial (Madero y Yacobaccio 1994; Yacobaccio et al. 1998). Para evaluar si estos resultados eran una función de la resistencia diferencial de los huesos a la atrición, aplicamos el análisis de correlación no-paramétrico $r$ de Spearman, utilizando los valores de densidad ósea publicados por Stahl (1999). Obtuvimos un resultado negativo $(-0,016528)$ y no significativo ( $p$. $<0,05,0,869691)$.

Descartada la destrucción mediada por densidad ósea procedimos a evaluar otros modelos, que permitan dar cuenta de la selección de partes como resultado de la conducta humana. Nuestro principal marco de referencia fue la anatomía económica de los camélidos, cuya utilidad reside en ser un factor objetivo que pudo influir sobre las elecciones de los actores, en situaciones como el transporte desde un sitio de matanza (Binford 1978) o el reparto de productos animales mediado por diferencias sociales. Siguiendo la sugerencia de Marean y Frey (1997), este análisis se realizó por separado para los esqueletos apendicular y axial. Se utilizaron los índices de utilidad alimenticia o FUI (Food Utility Index) para Lama glama, publicados por Mengoni (1991).

Para el esqueleto axial se obtuvo una correlación negativa $(r-0,729487)$ y significativa (p. 0,016647), mientras que para el esqueleto apendicular la correlación es positiva y no significativa ( $r 0,379536, p .0,313717)$. Estos resultados son contradictorios y difíciles de interpretar. Para empezar, cabe suponer que Soria 2 fue el locus de la preparación y consumo de los animales por la comunidad doméstica. Por lo tanto, la correlación negativa obtenida para el esqueleto axial es contraria a lo que podríamos esperar. Este fenómeno es lo que muchos denominan una curva inversa (reverse utility curve) (Lyman 1994; Marean y Frey 1997), que puede resultar de la destrucción mediada por densidad. Otra observación pertinente es que el valor FUI más alto corresponde a las costillas (Mengoni 1991), cuya identificabilidad taxonómica es muy baja en caso de estar fragmentadas. Otro problema a tener en cuenta es que, dado el escaso número de especímenes identificados al nivel de especie, el MAU debió calcularse para la familia Camelidae en su conjunto, de manera que no se discriminaron los aportes relativos de la caza y el pastoreo. Finalmente, es posible que el conjunto no sea una muestra representativa, dado que todavía falta excavar la mitad norte del Recinto 1.

Otro modelo que consideramos fue el "efecto ch'arki" descrito por Miller (1979). Este autor afirma que en los sitios de preparación de ch'arki estarían sobrerrepresentados 


\begin{tabular}{|c|c|c|}
\hline Taxón & $\begin{array}{l}\text { Tamaño } \\
\text { Corporal }\end{array}$ & Total NSP \\
\hline Artiodactyla & 4 & 334 \\
\hline Cervidae & 4 & 1 \\
\hline Hippocamelus antisensis & 4 & 1 \\
\hline Camelidae & 4 & 389 \\
\hline Lama sp. cf guanicoe cf glama & 4 & 3 \\
\hline Lama glama & 4 & 7 \\
\hline Lama guanicoe & 4 & 3 \\
\hline Vicugna vicugna & 4 & 2 \\
\hline Rodentia & 1 & 5 \\
\hline Caviidae & 1 & 1 \\
\hline Chinchillidae & $1-2$ & 3 \\
\hline Lagidium sp. & 2 & 11 \\
\hline Chaetophractus vellerosus & 2 & 5 \\
\hline Subtotal identificados (NISP) & & 766 \\
\hline No identificable & 1 & 2 \\
\hline No identificable & $1-2$ & 18 \\
\hline No identificable & 2 & 9 \\
\hline No identificable & $2-3$ & 23 \\
\hline No identificable & 3 & 14 \\
\hline No identificable & $3-4$ & 405 \\
\hline No identificable & 4 & 198 \\
\hline No identificable & 9 & 2443 \\
\hline TOTAL & & 3877 \\
\hline
\end{tabular}

Tabla 1. Composición de la muestra del Recinto 1 de Soria 2

Table 1. Soria 2, Structure 1, archaeofaunal assemblage.

los cráneos, metapodios y falanges, y que el patrón inverso correspondería a los lugares de consumo. También tuvimos en cuenta la revisión de este modelo y las alternativas propuestas por Stahl (1999). Ninguno de los perfiles hipotéticos derivados de estos modelos se correspondía con la frecuencia de elementos observada en Soria 2.

\section{Marcas de procesamiento}

El procesamiento de las carcasas puede resultar en la modificación intencional o accidental de los huesos. En cualquiera de los dos casos, los procedimientos técnicos implicados dependen de la anatomía del animal y del instrumental utilizado, por lo que las huellas de procesamiento permiten su inferencia.

En la figura 5 se indica la localización anatómica y la

\begin{tabular}{lcc}
\hline Taxón & $\begin{array}{c}\text { Tamaño } \\
\text { Corporal }\end{array}$ & NSP \\
\hline Artiodactyla & 4 & 23 \\
Camelidae ind. & 4 & 38 \\
Hippocamelus antisensis & 4 & 2 \\
Rodentia & 1 & 4 \\
& & \\
Chaetophractus vellerosus & 2 & 2 \\
& Subtotal & 69 \\
No identificado & 1 & 1 \\
No identificado & $1-2$ & 1 \\
No identificado & 2 & 4 \\
No identificado & $3-4$ & 31 \\
No identificado & 4 & 18 \\
No identificado & 9 & 47 \\
Total & & 171 \\
\hline
\end{tabular}

Tabla 2. Composición de la muestra del Recinto 2 de Soria 2

Table 2. Soria 2, Structure 2, archaeofaunal assemblage.

frecuencia de diferentes marcas, expresadas como NISP.

Esqueleto axial: los restos de mandíbula exhibían marcas de corte oblicuas y transversales sobre las caras medial y lateral de la rama, que de acuerdo a lo observado por Binford (1981) pudieron producirse durante el corte del músculo masetero y la remoción de la mandíbula. Una epífisis de vértebra cervical presenta otra huella de corte, que podría estar asociada a la separación de la cabeza. También hay marcas de corte sobre el íleon y el isquion, así como una marca de machacado sobre el segundo. Las primeras pueden resultar de la separación del sacro y la pelvis, mientras que las marcas sobre el isquion podrían haberse producido durante la separación de las patas traseras.

Esqueleto apendicular: hay marcas de corte y de machacado sobre fragmentos de húmero y radioulna, en sectores próximos a las articulaciones. También se observaron cortes sobre un astrágalo, un navicular, un cuboides y sobre la porción antero-proximal de un metatarso. Por su localización todas estas huellas debieron ocurrir durante el desmembramiento de las carcasas. Una rótula presenta un corte que pudo tener lugar durante la remoción de carne, ya que en ocasiones esta actividad deja marcas sobre la cara anterior de la diáfisis distal del fémur, justo sobre la superficie de la tróclea.

En resumen, hay bastantes evidencias de procesamiento y desarticulación (trozado primario y secundario). No hay signos de fileteado o remoción de los músculos, aunque debemos tener presente que las marcas de procesamiento son un efecto secundario influido por factores como la 


\begin{tabular}{|c|c|c|c|c|c|c|c|c|c|c|c|c|c|c|}
\hline \multirow{3}{*}{ Elemento } & \multirow{3}{*}{ NISP } & \multicolumn{9}{|c|}{ MNE } & \multirow{3}{*}{$\begin{array}{l}\text { MNE } \\
\text { final }\end{array}$} & \multirow{3}{*}{$\mathrm{MNI}$} & \multirow{3}{*}{ MAU } & \multirow{3}{*}{$\% \mathrm{MAU}$} \\
\hline & & \multicolumn{3}{|c|}{ Izquierdo } & \multicolumn{3}{|c|}{ Derecho } & \multicolumn{3}{|c|}{ S/D o Axial } & & & & \\
\hline & & $A$ & SA & ind & $A$ & SA & ind & A & SA & ind & & & & \\
\hline Cráneo & 14 & - & - & - & - & - & - & 0 & 1 & 2 & 3 & 3 & 3,00 & 50,00 \\
\hline Mandíbula & 8 & - & - & - & - & - & - & 3 & 0 & 0 & 3 & 3 & 3,00 & 50,00 \\
\hline Dientes sueltos & 17 & - & - & - & - & - & - & - & - & - & - & - & - & - \\
\hline V. Atlas & 1 & - & - & - & - & - & - & 0 & 0 & 1 & 1 & 1 & 1,00 & 16,67 \\
\hline V. Axis & 2 & - & - & - & - & - & - & 0 & 2 & 0 & 2 & 2 & 2,00 & 33,33 \\
\hline V. Cervicales 3-7 & 41 & - & - & - & - & - & - & 2 & 5 & 3 & 10 & 2 & 2,00 & 33,33 \\
\hline V. Torácicas & 11 & - & - & - & - & - & - & 1 & 6 & 1 & 8 & 2 & 0,67 & 11,11 \\
\hline Costillas & 14 & 4 & - & - & 6 & - & - & 4 & - & - & 14 & 1 & 0,58 & 9,72 \\
\hline V. Lumbares & 7 & - & - & - & - & - & - & 2 & 2 & 0 & 4 & 2 & 0,57 & 9,52 \\
\hline Sacro & 0 & - & - & - & - & - & - & 0 & 0 & 0 & 0 & 0 & 0,00 & 0,00 \\
\hline Escápula & 7 & - & 1 & 2 & - & 1 & 3 & - & - & - & 7 & 4 & 3,50 & 58,33 \\
\hline Húmero & 20 & - & 1 & 5 & - & 1 & 2 & - & - & - & 9 & 6 & 4,50 & 75,00 \\
\hline Radioulna & 20 & 1 & 2 & - & - & 6 & & - & - & - & 9 & 7 & 4,50 & 75,00 \\
\hline Escafoides & 4 & - & - & 1 & - & - & 3 & - & - & - & 4 & 3 & 2,00 & 33,33 \\
\hline Lunar & 2 & - & - & 1 & - & - & 1 & - & - & - & 2 & 1 & 1,00 & 16,67 \\
\hline Cuneiforme & 2 & - & - & 1 & - & - & 1 & - & - & - & 2 & 1 & 1,00 & 16,67 \\
\hline Mágnum & 2 & - & - & & - & - & 2 & - & - & - & 2 & 2 & 1,00 & 16,67 \\
\hline Unciforme & 10 & - & - & 4 & - & - & 5 & - & - & - & 9 & 5 & 4,50 & 75,00 \\
\hline Trapezoides & 4 & - & - & 3 & - & - & 1 & - & - & - & 4 & 3 & 2,00 & 33,33 \\
\hline Pisciforme & 1 & - & - & & - & - & 1 & - & - & - & 1 & 1 & 0,50 & 8,33 \\
\hline \multirow[t]{2}{*}{ Metacarpo } & 9 & - & - & 3 & - & - & 2 & - & - & - & 5 & 3 & 2,50 & 41,67 \\
\hline & & & & & & & & & & & & & & 0,00 \\
\hline Innominado & 24 & - & 2 & 4 & - & - & 6 & - & - & - & 12 & 6 & 6,00 & 100,00 \\
\hline Fémur & 16 & - & 2 & 1 & 1 & 1 & - & - & - & - & 5 & 3 & 2,50 & 41,67 \\
\hline Rótula & 6 & - & - & - & - & - & 5 & - & - & - & 6 & 5 & 3,00 & 50,00 \\
\hline Tibia & 14 & - & 2 & 2 & 1 & 1 & 2 & - & - & - & 8 & 4 & 4,00 & 66,67 \\
\hline Astrágalo & 8 & - & - & 2 & - & - & 6 & - & - & - & 8 & 6 & 4,00 & 66,67 \\
\hline Calcáneo & 8 & 2 & - & - & - & 2 & 1 & - & - & - & 8 & 5 & 4,00 & 66,67 \\
\hline Navicular & 8 & - & - & 5 & - & - & 3 & - & - & - & 8 & 5 & 4,00 & 66,67 \\
\hline Cuboides & 6 & - & - & 4 & - & - & 2 & - & - & - & 6 & 4 & 3,00 & 50,00 \\
\hline Maléolo lateral & 10 & - & - & 4 & - & - & 6 & - & - & - & 10 & 6 & 5,00 & 83,33 \\
\hline Metatarso & 6 & - & - & 2 & - & - & 3 & - & - & - & 5 & 3 & 2,50 & 41,67 \\
\hline Metapodios & 25 & - & - & - & - & - & - & 3 & 4 & - & 7 & 2 & 1,75 & 29,17 \\
\hline Sesamoideos & 4 & - & - & - & - & - & - & - & - & 4 & 4 & 1 & 0,25 & 4,17 \\
\hline Falange 1 & 32 & - & - & - & - & - & - & 9 & 9 & - & 18 & 4 & 2,25 & 37,50 \\
\hline Falange 2 & 27 & - & - & - & - & - & - & 13 & 7 & - & 20 & 3 & 2,50 & 41,67 \\
\hline Falange 3 & 14 & - & - & - & - & - & - & - & - & 14 & 14 & 2 & 1,75 & 29,17 \\
\hline
\end{tabular}

Tabla 3. Frecuencia de elementos anatómicos, expresada como número de especímenes identificados por elemento, mínimo número de elementos (MNE), mínimo número de individuos (MNI), mínimo de unidades anatómicas y mínimo de unidades anatómicas estandarizado (MAU y MAU\%).

Table3. Anatomical elements frequency, expressed as number of identified specimens by element, minimal number of elements (MNE), minimal number of individuals (MNI), minimal anatomical units and standardized minimal anatomical units (MAU and \%MAU) 


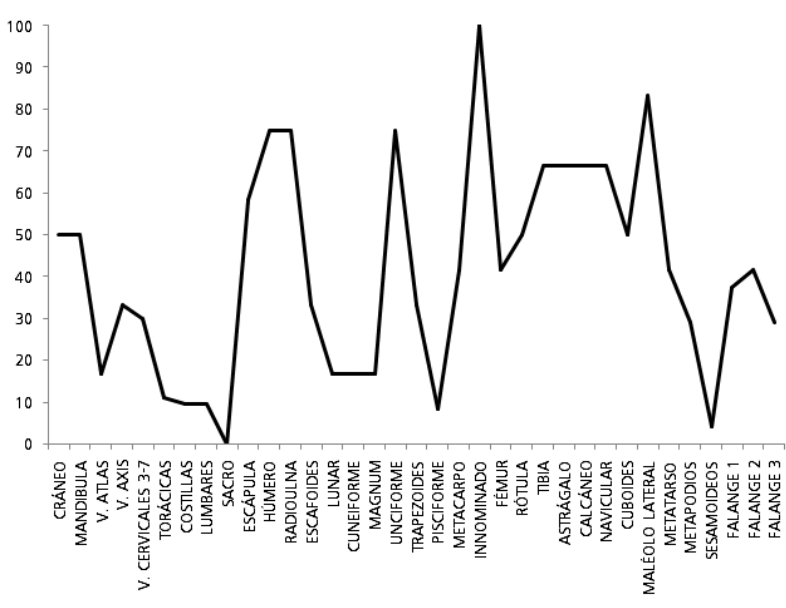

Figura 4. Frecuencia de partes esqueletarías (\%MAU) del subconjunto Camelidae de Soria 2, Recinto 1.

Figure 4. Skeletal parts frequency (\%MAU). Camelids from Soria 2, Structure 1.

dureza y filo de los instrumentos, o la destreza del operario (Dewbury y Russell 2007; Lyman 2005).

Fragmentación del conjunto y patrones de fractura en huesos largos

Los restos de camélido presentan un alto grado de fragmentación. Si se omiten dientes sueltos y sesamoideos, el número de huesos completos asciende a 100, en su mayoría carpos, tarsos y falanges mediales y distales. Para el estudio de las fracturas nos limitamos a los fragmentos de huesos con cavidad medular (huesos largos, calcáneo y falanges primera y segunda). Su longitud media es 55, $16 \mathrm{~mm}$, con un Desvío Estándar de 28,98 mm. De los especímenes para los cuales pudo identificarse el estado del hueso al momento de su rotura, 41 estaban frescos y 35 estaban secos. Unos 16 fragmentos presentaban negativos o muescas de impacto sobre las diafisis. La fractura intencional de los huesos pudo tener por fin la obtención de médula, su procesamiento para la obtención de grasa ósea o la manufactura de instrumentos (Belotti 2010b).

\section{Termoalteración}

Para analizar la termoalteración del conjunto se registró la coloración de los huesos y la presencia de alteraciones superficiales (Nicholson 1993; Mengoni 1999). En la tabla 4 se presenta el número de especímenes discriminados según su grado de termoalteración. Casi la mitad del conjunto (NISP 199) presenta alguna evidencia de combustión. Además de los cambios en la coloración y la textura, unos 12 especímenes presentan evidencias adicionales como cuarteaduras y exfoliaciones. Los huesos pudieron quemarse debido a su utilización como combustible o durante la preparación de alimentos. También pudo ser un resultado no intencional del descarte.

\begin{tabular}{cccccc}
\hline Color & 0 & 1 & 2 & 3 & Total \\
NISP & 205 & 127 & 49 & 23 & 404 \\
\hline
\end{tabular}

Tabla 4. Número de especímenes con o sin evidencias de termoalteración. 0. Sin quemar. 1 Quemado. 2. Carbonizado. 3. Calcinado.

Table 4. Frequency of thermoaltered and not modified bones. 0. unburned. 1 burned. 2. carbonized. 3. calcinated.

\section{Discusión y conclusiones}

Retomando las hipótesis sobre la FES tribal, pensamos que el grueso de la producción era llevado adelante por el grupo familiar y para la satisfacción directa de sus necesidades (Belotti 2007). Desde una perspectiva arqueológica cabría esperar que el espacio doméstico fuera el locus de un amplio rango de actividades productivas y reproductivas, como la fabricación, uso y mantenimiento de medios de trabajo, y la preparación y consumo de alimentos.

En lo que respecta a la evidencia faunística, esperaríamos que el registro se asemeje al producido por grupos domésticos actuales dedicados al pastoreo (e. g. Madero y Yacobaccio 1994; Yacobaccio et al. 1998). Dado que se presume la naturaleza igualitaria de las sociedades tribales tempranas, en una comparación con sitios coetáneos no deberían registrarse diferencias en el acceso a recursos faunísticos. Sin embargo esta comparación no es sencilla, en parte debido a las diferentes historias tafonómicas de cada sitio. Pero además, el acceso a los animales no depende únicamente de las relaciones de producción, sino también de la división del trabajo, las tecnologías de transporte y conservación, de las instituciones que organizan el intercambio, etcétera. Aún en las sociedades feudales y capitalistas, existen subgrupos de las clases explotadas que tienen acceso a animales enteros por ser productores directos o por controlar sus medios de producción (e. g. el campesinado). Entonces, para establecer indicadores zooarqueológicos de desigualdad es necesario incorporar diversas variables contextuales y tener un marco de referencia adecuado. Por ejemplo, en muchas sociedades clasistas premodernas la distancia social se expresa en un acceso diferencial a ciertos recursos, como sucedía con la prohibición de la caza para los campesinos medievales.

Finalmente, son necesarias otras líneas de evidencia para lograr reconstrucción bien fundamentada de los sistemas sociales prehistóricos. Frente a esta dificultad, de momento no podemos hacer otra cosa que esperar a que algunas de las ideas aquí vertidas sean retomadas por otros investigadores.

El piso de ocupación del Recinto 1 se caracteriza por una alta densidad de hallazgos. Estimando su volumen a partir de la superficie excavada y su potencia media, 


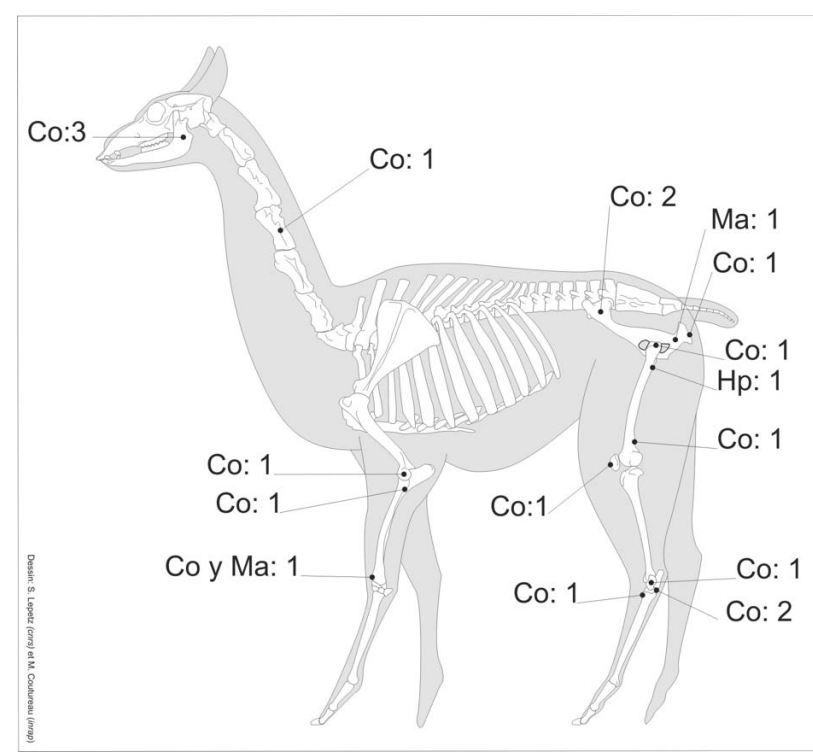

Figura 5. Localización y frecuencia de marcas de procesamiento, expresada como número de especímenes identificados (NISP). Modificado de imagen original por S. Lepetz y M. Coutureau (fuente http://www.archeozoo.org).

Figure 5. Location and processing marks frequency, expressed as number of specified specimens (NISP). Modified from S. Lepetz and M. Coutureau (http://www.archeozoo.org).

por cada $\mathrm{dm}^{3}$ de sedimento tenemos en promedio 0,907 fragmentos de cerámica, 0,08 especímenes óseos identificables y 0,104 artefactos líticos. La posición horizontal de la mayoría de los tiestos y en algunos casos su fractura in situ indican un contexto de depositación primaria, posiblemente como desechos de facto (Spano 2008).

En los últimos dos años varios trabajos de tesis avanzaron considerablemente en el estudio de los componentes arqueológicos del sitio Soria 2. Sus resultados permitieron formular hipótesis sobre las actividades que pudieron llevarse a cabo en el sitio. La reconstrucción del repertorio morfológico de la cerámica ordinaria permitió reconocer piezas que pudieron servir para el almacenamiento de sólidos y líquidos (ollas), cocción de alimentos (ollas con hollín), procesamiento sin calor (cuencos y escudillas grandes), transporte y servicio de líquidos (jarras pequeñas) y de comida (cuencos y escudillas), a lo que debemos sumar los restos de cinco cucharas (Baigorria Di Scala 2009). En el caso de la cerámica fina se observó un predominio de formas abiertas, pucos sobre todo, que debieron utilizarse para servir alimentos (Spano 2008). El subconjunto fino incluía también botellones, vasos y cucharas. En el pozo de basura de la esquina sudeste se recuperó un hornillo de pipa, que presenta evidencias de haber sido empleado para el consumo de psicoactivos (Rosso y Spano 2005-2006).

La abundancia de desechos de talla indica que parte del trabajo sobre piedra tenía lugar en el espacio doméstico. Según Carbonelli (2009) la actividad predominante pudo ser la formatización de filos. Entre los instrumentos se recuperaron raederas, raspadores, cuchillos, artefactos de retoque sumario, filos naturales, puntas de proyectil e instrumentos de molienda (manos y morteros). Pensamos que los instrumentos con filo pudieron usarse durante el procesamiento de las carcasas animales.

Los restos de fauna se vinculan directamente a la alimentación, pero además permiten inferir otras actividades. Respecto a la composición taxonómica del conjunto, hay un predominio absoluto de la familia Camelidae y de artiodáctilos indeterminados. Esto es una tendencia común a los sitios agroalfareros del NOA y de Yocavil, (Olivera 1997, Izeta 2007). Hacia fines del primer milenio y luego durante los Desarrollos Regionales ocurriría una leve diversificación de la dieta en los valles (Izeta 2007, Pratolongo 2008, Belotti 2010a).

Para Camelidae están representados casi todos los elementos anatómicos, aunque no en las proporciones que cabría esperar en los animales vivos. Las diferencias pueden obedecer a distintas determinaciones, como el transporte selectivo, el reparto entre unidades domésticas ${ }^{2}$, la superposición de los productos de caza y pastoreo, entre otras. Como vimos antes, la comparación entre el perfil anatómico arqueológico y las expectativas derivadas de distintos marcos de referencia y modelos resulta problemática. Esto no debería sorprender dada la complejidad de los procesos tafonómicos y las diferencias de identificabilidad entre especímenes óseos. Como ejemplo, recordemos que en algunos casos la aplicación de análisis de conglomerados puede agrupar por separado los perfiles anatómicos derivados de la etnoarqueología, respecto de los casos arqueológicos (e. g. Madero y Yacobaccio 1994, Mengoni 1999, Belotti 2007). Aun así cabe la posibilidad de que algunos ejemplares ingresaran casi enteros a la unidad residencial. Por ejemplo si los animales domésticos se sacrificaban en las inmediaciones.

El trozado de las carcasas se deduce de la desorganización anatómica del conjunto y por la presencia de marcas de corte cerca de las articulaciones de los huesos largos. Asimismo, todos los huesos estaban muy fragmentados, quizá para obtener nutrientes como médula, seso y grasa ósea (Belotti 2010b).

La identificación de L. guanicoe y $V$. vicugna implica que la caza mantuvo su importancia, a la par del pastoreo. Para otros sitios del sur de los Valles Calchaquíes, las investigaciones de Izeta (2007) demuestran ampliamente la complementariedad de ambas actividades durante el primer milenio. En algunos sitios formativos de la

\footnotetext{
${ }^{2}$ Aunque la FES y el Modo de Producción tribal suponen cierta autosuficiencia de la comunidades domésticas, esto no significa que no hubiera entre ellas algún intercambio. De hecho, diversas formas de reciprocidad permiten movilizar excedentes agropecuarios o de fuerza de trabajo entre las unidades domésticas vinculadas por consanguineidad o afinidad (e. g. Vargas 1987)
} 
Puna incluso se documentó el predominio de especies silvestres (e. g. Elkin 1996; Haber 2001). Una posible explicación es que la caza sirviera como una forma de diversificación económica orientada a amortiguar el riesgo de stress ambiental (Göbel 1994). Entre pastores actuales esto se logra con la cría de rebaños de diferentes especies y familias (e.g. Yacobaccio et al. 1998), lo que era impracticable en el NOA prehispánico con una sola especie de ungulados domésticos. La cacería de camélidos silvestres pudo ocurrir dentro de un patrón de movilidad estacional asociado al pastoreo (Olivera 1997, 2001).

Un problema importante es el de la procedencia de los restos asignados a $V$. vicugna. La distribución actual de la vicuña se restringe a elevaciones superiores a los 3500 msnm (Vilá 2006). Si este fuera también el caso hace 2000 años, podríamos pensar que estos animales fueron capturados en la Puna. La explotación directa o indirecta del ambiente puneño está atestiguada por la presencia de artefactos de obsidiana en Soria 2 (puntas de proyectil, dos núcleos y desechos de talla), así como por algunas modalidades decorativas en la cerámica que remiten a la puna (Spano 2008). Una de las principales contradicciones de la FES tribal se origina en los diferentes recursos naturales disponibles en el territorio de cada comunidad aldeana, lo que promueve estrategias orientadas a la explotación de múltiples entornos. De manera directa como en el control de un máximo de pisos ecológicos o el sedentarismo dinámico, o indirectamente a través del intercambio con grupos de la puna, con la intermediación de caravaneros por ejemplo. Cabe también la posibilidad de que en ese tiempo fueran animales locales, o que tuvieran una distribución más amplia y llegaran al valle del Cajón. Para Ambato durante el Período Medio o de Integración Regional, Pérez Gollán (2000) propuso que pudieron cazarse vicuñas en los altos de Singuil, a solo $1500 \mathrm{msnm}$.

Soria 2 presenta similitudes y diferencias con los sitios formativos del sur de los valles Calchaquíes estudiados por Izeta (2007). A fin de detectar tendencias diacrónicas en el registro zooarqueológico, Izeta dividió el primer milenio D.C. en cuatro bloques temporales. Aquí incluiremos a Soria 2 en el Bloque Temporal 1 (ca. 1900-1700 A.P.), junto al depósito $\mathrm{VI}$ de Bañado Viejo y las estructuras 3 y 11 de Yutopian. Tomados en conjunto, los tres contextos estudiados por Izeta muestran una explotación pareja de especies silvestres y domésticas (L. glama MNI 2, L. guanicoe $\mathrm{MNI} 1$, V. vicugna $\mathrm{MNI} 1$, Lama sp. MNI 8), y un leve predominio de los animales adultos, con un MNI de siete individuos, contra cinco juveniles (Izeta 2007). Individualmente presentan cierta variación, pero en general permiten llegar a las mismas conclusiones. Ambas tendencias se mantienen en los bloques posteriores, con una preponderancia levemente mayor de animales adultos (MNImx\% 70) hacia el Bloque 4 (1100-1000 AP).

Cuando incorporamos los datos de Soria 2 al Bloque 1, la relación entre especies silvestres y domésticas cambia a favor de las primeras (L. glama MNI 3, L. guanicoe $\mathrm{MNI} 3$, V. vicugna $\mathrm{MNI} 2$ ). Esto puede representar una insuficiencia de la técnica pecuaria entre las primeras sociedades aldeanas de Yocavil, aunque debemos observar que los especímenes identificados al nivel de especie representan una muestra muy pequeña de cada conjunto, y que el análisis morfométrico no es aplicable a los camélidos subadultos.

Un cambio similar tiene lugar en la relación entre animales adultos y subadultos. En las dos estructuras de Yutopian el MNI de camélidos adultos fue tres y el de juveniles dos, mientras que en Bañado Viejo ambas clases de edad tienen un MNI igual a uno. En Soria ocurre todo lo contrario (MNI adultos 4, MNI subadultos 6). Cuando se incorpora Soria al Bloque 1, el MNI de subadultos pasa de representar un $30 \%$ de los individuos estimados a un $50 \%$.

Hay varias causas posibles para una mayor presencia de subadultos, como el efecto de enfermedades de corral que afligen a los neonatos y juveniles, por ejemplo la enterotoxemia o fiebre intestinal (Sotomayor Berrío 1984), o ciertas prácticas mágicas (Flores Ochoa 1975). Un patrón semejante se repite en otros sitios formativos como Campo Colorado en el valle Calchaquí (Salta) y los sitios puneños Casa Chávez Montículos y Real Grande, lo cual según Olivera sería el resultado de una estrategia de matanza selectiva en un sistema ganadero poco desarrollado (rebaños pequeños, ausencia de pasturas cultivadas) (Olivera 1997, 2001). Esta situación habría cambiado hacia el año 1000 A.P., gracias a innovaciones tecnológicas como el cultivo de forraje (Olivera 1997). Esta hipótesis nos parece válida para Soria 2 por su cronología temprana, e incluso pensamos que las tendencias observadas en el sitio podrían reflejar un Período de ajuste o perfeccionamiento en la explotación de los recursos pecuarios, aunque al presente no estamos en condiciones de poner a prueba esta idea.

\section{Palabras finales}

La adecuación de las hipótesis sobre la FES tribal para el Formativo de Yocavil no puede corroborarse solo con la evidencia faunística de un sitio. Nuestra intención aquí es más bien explorar una serie de interpretaciones posibles sobre los resultados obtenidos, que a futuro puedan ser contrastadas con una base de datos más amplia. Desde el año 2007 venimos analizando los materiales faunísticos de diversos sitios de los valles de Yocavil y Calchaquí, con fechados que se extienden desde los albores del primer milenio y hasta la conquista incaica del NOA, a lo que debemos sumar la información disponible en la bibliografía (e. g. Izeta 2007, Pratolongo 2008). En un trabajo reciente intentamos además formular y contrastar algunas hipótesis relativas a la intensificación de la subsistencia en el contexto de una creciente complejidad 
social (Belotti 2010a). Esperamos en el futuro cercano poder presentar un panorama más completo.

Buenos Aires, 2 de febrero de 2011

\section{Agradecimientos}

Los trabajos de excavación y de laboratorio se llevaron adelante en el marco de los subsidios UBACyT F-152, CONICET PIP 6148, FONCYT PICT 12163 y 34511.

El presente trabajo es producto de mi tesis de licenciatura, por lo que mi primer agradecimiento es para mis directores, Myriam Tarragó y Andrés D. Izeta, así como para mis evaluadores Daniel E. Olivera y María Isabel González de Bonaveri. También a Valeria Palamarczuk, Romina Spano, Luis González y en general a todos mis compañeros y amigos del proyecto Yocavil, y profesores y amigos del seminario anual de investigación.

\section{Bibliografía}

Albeck, M. E. 2000. La Vida Agraria de los Andes del Sur. M. Tarragó (Ed.) Los Pueblos Originarios y la Conquista pp 187-228, Sudamericana, Buenos Aires.

Álvarez Larrain, A., J. Baigorría di Scala, C. R. Belotti López de Medina, J. P. Carbonelli, S. Grimoldi, M. S. López, D. Magnifico, V. Palamarczuck, R. Spano, G. Spengler, L. Stern Gelman y F. Weber. 2009. Avances en el estudio de un contexto doméstico formativo en el valle de Yocavil. T. Bourlot, D. Bozzuto, C. Crespo, A. C. Hecht y N. Kuperszmit (Eds.), Entre pasados y presentes II. Estudios contemporáneos en ciencias Antropológicas: 369-382, FHNFA, Buenos Aires.

Bate, L. F. 1998. El Proceso de Investigación en Arqueología. Crítica, Barcelona.

Baigorria Di Scala, J. 2009. El sitio Formativo Soria 2: Estudio Tecno-Morfológico del conjunto cerámico ordinario. Tesis de licenciatura inédita. Facultad de Filosofía y Letras, Universidad de Buenos Aires, Argentina,

Baigorria Di Scala, J. y R. Spano 2007. Análisis preliminar de la cerámica del sitio formativo Soria 2 (Valle de Yocavil, Catamarca). XVI Congreso Nacional de Arqueología Argentina Tomo III: 65-70. EdiUnju, San Salvador de Jujuy.

Belotti López de Medina, C. R. 2007. Zooarqueología del sitio Soria 2 (Departamento de Santa María, Provincia de Catamarca) y estudio comparativo del registro zooarqueológico del sur de los valles Calchaquíes, para Ios Períodos Formativo y Desarrollos Regionales (siglos I A. C. $a X V D$. C.). Facultad de Filosofía y Letras, Universidad de Buenos Aires, Argentina.

Belotti López de Medina, C. R. 2010a. Una primera aproximación al desarrollo del modo de producción tribal y la evolución del registro zooarqueológico en el sur de los valles Calchaquíes (Catamarca). M. A. Gutiérrez, M. De Nigris, P. M. Fernández, M. Giardina, A. Gil, A. Izeta, G. Neme y $\mathrm{H}$. Yacobaccio (Eds.), Zooarqueología a principios del siglo XXI, Aportes teóricos, metodológicos y casos de estudio: 189-198, Ediciones del Espinillo, Buenos Aires.

Belotti López de Medina, C. R. 2010b. Rompiendo huesos para el puchero. Análisis de la fragmentación de un conjunto arqueofaunístico del Período Formativo del valle de Yocavil. La arqueometría en Argentina y Latinoamérica pp 219-224 FFyH-UNC, Córdoba.

Behrensmeyer, A. K. 1978. Taphonomic and ecologic information from bone weathering. Paleobiology 1:150162.

Benavente, M. A., L. Adaro, P. Gecele y C. Cunazza, 1993. Contribución a la determinación de especies animales en arqueología: familia Camelidae y taruca del norte. Andes y Andes S. A., Santiago de Chile.

Bennett, W. C. 1948. Northwest Argentine Archaeology, Yale University Publications in Anthropology, New Haven.

Binford, L. R. 1978. Nunamiut Ethnoarchaeology. Academic Press, New York.

Binford, L. R.1981. Bones: ancient men and modern myths. Academic Press Inc., New York.

Carbonelli, J. P. 2009."Interacciones cotidianas entre materias primas y sujetos sociales en el Valle de Yocavil. El caso del sitio Soria 2 (Andalhuala, Pcia. de Catamarca". Facultad de Filosofía y Letras, Universidad de Buenos Aires, Argentina. 217 páginas. Museo Etnográfico.

Dewbury A. G. y N. Russell 2007. Relative frequency of butchering cutmarks produced by obsidian and flint: an experimental approach. Journal of Archaelogical Science 34: 354-357

Elkin, D. 1986. "Arqueozoología de Quebrada Seca 3: indicadores de subsistencia humana temprana en la Puna Meridional Argentin". Facultad de Filosofía y Letras, Universidad de Buenos Aires.

Flores Ochoa, J. A. 1975. Pastores de alpacas. Allpanchis 8 (8): 5-23

Göbel, B. 1994. El Manejo del Riesgo en la Economía Pastoril de Susques. D. E. Elkin, C. M. Madero, G. L. Mengoni, D. E. Olivera, M. C. Reigadas y H. D. Yacobaccio (Eds.), Zooarqueología de Camélido. 1: 43-56, GZC, Buenos Aires.

González, A. R. 1979. Dinámica cultural del N.O. argentino. Evolución e historia en las culturas del N.O. 
Argentino. Antiquitas 28-29: 1-15.

González, A. R. 1998. Cultura La Aguada. Arqueología y Diseños. Filmediciones, Buenos Aires.

Gonzáles, A. R., J. A. Pérez. 1966. El área andina meridional. XXXVI Congreso Internacional de Americanista. I: 241265, Sevilla.

Haber, A. F. 2001. El oasis en la articulación del espacio circumpuneño. Actas del XIII Congreso Nacional de Arqueología Argentina, Tomo 1: 251-267, Córdoba.

Izeta, A. D. 2007. Zooarqueología del Sur de los Valles Calchaquíes (Provincias de Catamarca y Tucumán, República Argentina). British Archaeological Reports, Oxford.

Izeta, A. D., M. C. Scattolin. 2001. Bañado Viejo: análisis faunístico de un sitio formativo en el fondo del valle de Santa María. En Actas del XIII Congreso Nacional de Arqueología Argentina, Vol. 2, pp. 385-395. Córdoba.

Kent, J. D. 1982." The Domestication and exploitation of the South American camelids: methods of analysis and their application to circum-lacustrine archaeological sites in Bolivia and Per", Washington University, St. Louis, Missouri, USA.

Lyman, R. L. 1994. Vertebrate Taphonomy. Cambridge University Press, Cambridge.

Lyman, R. L. 2005. Analyzing cut marks: lessons from artiodactyl remains in the northwestern United States. Journal of Archaeological Science 32: 1722-1732.

Madero, C. M., H. D. Yacobaccio. 1994. El registro faunístico del pastoreo actual y sus implicancias arqueológicas. D. E. Elkin, C. M. Madero, G. L. Mengoni, D. E. Olivera, M. C. Reigadas y H. D. Yacobaccio (Eds.), Zooarqueología de Camélido. 1: 73-94, GZC, Buenos Aires.

Marean, C. W., C. J. Frey. 1997. Animal bones from caves to cities: reverse utility curves as methodological artifacts. American Antiquity 62 (4): 698-711

Meillassoux, C. 1977. Mujeres, graneros y capitales: economía doméstica y capitalismo. Siglo XXI, México.

Menegaz, A., M. Salemme y E. Ortiz Jaureguizar. 1988. Una propuesta de sistematización de los caracteres morfométricos de los metapodios y las falanges de camelidae. N. Ratto y A. Haber (Eds.), De Procesos, Contextos y otros Huesos, :pp 53-64, Instituto de Ciencias Antropológicas (FFyL-UBA), Buenos Aires.

Mengoni Goñalons, G. L. 1991. La llama y sus productos primarios. Arqueología 1: 179-196.

Mengoni Goñalons, G. L. 1999. Cazadores de Guanacos de la estepa patagónica. Sociedad Argentina de Antropología, Buenos Aires.

Miller, G. R. 1979."An introduction to the ethnoarchaeology of andean camelid", University of California, Berkeley.

Nicholson, R. A. 1993. A Morphological Investigation of Burnt Animal Bone and an Evaluation of its Utility in Archaeology. Journal of Archaeological Science 20: 411-428.

Núñez Regueiro, V. A. 1974. Conceptos instrumentales y marco teórico en relación al análisis del desarrollo cultural del Noroeste Argentino. Revista del Instituto de Antropología 5: 169-190.

Núñez Regueiro, V. A. y M. R. Tartusi 2002. Aguada y el proceso de integración regional. Estudios Atacameños 24: 9-19.

Olivera, D. E. 1997. La importancia del recurso Camelidae en la Puna de Atacama entre los 10.000 y 500 años AP. Estudios Atacameños 14: 29-41.

Olivera, D. E. 2001. Sociedades agropastoriles tempranas: el formativo inferior del noroeste Argentino. E. Berberian y A. Nielsen (Eds.), Historia Argentina Prehispánica, Vol. 1: 83-125, Editorial Brujas, Córdoba.

Pacheco Torres, V. R., A. Altamirano Enciso y E. Guerra Porras. 1979. Guía osteológica para camélidos sudamericanos. Departamento Académico de Ciencias Histórico Sociales, Universidad Mayor de San Marcos, Lima.

Palamarczuk, V., R. Spano, D. Magnifico, F. Weber, S. López, y M. Manasiewicz. 2007. Soria 2. Apuntes sobre un sitio temprano en el valle de Yocavil (Catamarca, Argentina). Intersecciones en antropología 8: 121-135

Pratolongo, G. J. 2008. Estudio de los restos faunísticos de dos sitios tardíos del valle de Yocavil, provincia de Catamarca: Rincón Chico 15 y las Mojarras 1. M. N. Tarragó y L. R. González (Eds.), Estudios arqueológicos en Yocavil: 81-126, Asociación Amigos del Museo Etnográfico, Buenos Aires.

Pérez Gollán, J. A. 2000. El jaguar en llamas (la religión en el antiguo Noroeste argentino). M. Tarragó (Ed.), Los Pueblos Originarios y la Conquista: 229-256, Sudamericana, Buenos Aires.

Raffino, R. 1990. Poblaciones indígenas en Argentina. Tipográfica Editora Argentina, Buenos Aires. 
Rosso, C. y R. Spano 2005-2006. Humos del vecino: evidencias del uso de alucinógenos en pipas halladas en dos sitios tempranos de los Valles Calchaquíes. Arqueología 13: 79-98

Sarmiento Fradera, G. 1986. La sociedad cacical agrícola: hipótesis y uso de indicadores arqueológicos. Boletín de antropología americana 13: 35-64.

Scattolin, M. C. 2000. Santa María durante el Primer Milenio A.D. ¿Tierra Baldía?. Árstryck 1995-1998. Etnograsfika Museet i Goteborg: 63-83.

Scattolin, M. C. 2001. Organización residencial y arquitectura en el Aconquija durante el primer milenio A. D. En Actas del XIII Congreso Nacional de Arqueología Argentina, Vol. 1: 439-449, Córdoba.

Scattolin, M. C. 2006. De las comunidades aldeanas a los curacazgos en el Noroeste Argentino. Boletín de Arqueología PUCP 10: 357-398.

Scattolin, M. C. 2007. Santa Maria antes del año mil. Fechas y materiales para una historia cultural. V. I. Williams, B. N. Ventura, A. B. M. Callegari y H. D. Yacobaccio (Eds.), Sociedades Precolombinas Surandinas: Temporalidad, Interacción y Dinámica cultural del NOA en el ámbito de los Andes Centro-Sur: 203-219 Artes Gráficas Buschi, Buenos Aires.

Sotomayor Berrío, M. A. 1984. La explotación ganadera en las comunidades campesinas. Allpanchis XX (23): 97-103

Spano, R. 2008. "Indagaciones sobre las sociedades aldeanas del Valle de Yocavil; análisis de la alfarería fina del sitio Soria 2 (Andalhuala, pcia. de Catamarca)". Facultad de Filosofía y Letras, Universidad de Buenos Aires, Argentina. 182 páginas. Museo Etnográfico.

Stahl, P. W. 1999. Structural Density of Domesticated South American Camelid Skeletal Elements and the Archaeological Investigation of Prehistoric Andean Ch'Arki. Journal of Archaeological Science 26: 1347-1368

Statsoft. 2004. Electronic Statistics Textbook. mhttp//:www.statsoft.com. (última consulta 1/04/2007).

Tarragó, M. N. 1992. El Formativo y el Surgimiento de la Complejidad Social en el Noroeste Argentino. Simposio Internacional "Arqueología Suramericana. Una Reevaluación del Formativo", Cuenca, Ecuador. MS.

Tarragó, M. N. 1999. Las sociedades del sudeste andino.
En Historia General de América Latina, Vol. 1, pp. 465480. UNESCO París, Editorial Trotta, España.

Tarragó, M. N. 2000. Chacras y pukara. Desarrollos sociales tardíos. M. Tarragó (Ed.) Los Pueblos Originarios y la Conquista: 256-300, Sudamericana, Buenos Aires

Tarragó, M. N. y M. C. Scattolin 1999. La Problemátic del Período Formativo en el Valle de Santa Maria. En Actas del XII Congreso Nacional de Arqueología Argentina, Vol. 1, pp. 142-153. La Plata.

Tartusi, M. R. A., V. A. Núñez Regueiro. 1993. Los centros ceremoniales del NOA. Publicaciones del Instituto de Arqueología de la UNT, Serie Ensayo y Crítica, Tucumán.

Tartusi, M. R. A., V. A. Núñez Regueiro. 2001. Fenómenos cúlticos tempranos en la subregión valliserrana. E. Berberian y A. Nielsen (Eds.), Historia Argentina Prehispánica, Vol. 1: 127-170, Editorial Brujas, Córdoba.

Trigger, B.G. 1991. Constraint and Freedom. A New Sintesys for the Archaeological Explanation. American Anthropologist 93: 551-569

Vargas Arenas, I. 1985. Modo de vida: categoría de las mediaciones entre formación social y cultura. Boletín de antropología americana 12: 5-16.

Vargas Arenas, I. 1987. La formación económico social tribal. Boletín de antropología americana 15:15-26.

Vilá, B. 2006. Suborden Tylopoda, Familia Camelidae. R. M. Barquez, M. M. Díaz y R. A. Ojeda (Eds.), Mamíferos de Argentina. Sistemática y distribución: 116-118, SAREM, Tucumán.

Weber Sanguinetti, F. 2006. Informe de Campaña (2002, 2004 y 2005). Soria 2 - Andalhuala La Banda, Valle de Santa María, Catamarca. Facultad de Filosofía y Letras, Universidad de Buenos Aires. MS.

Wheeler, J. C. 1982. Aging Llamas and Alpacas by their Teeth. Llama World 1 (2): 12-17.

Wolf, E. 1987. Europa y la gente sin historia. Fondo de Cultura Económica, Buenos Aires.

Wolf, E. 1990. Facing Power. Old Insights, New Questions. American Anthropologist 92 (3): 586-596

Yacobaccio, H. D., C. M. Madero y M. P. Malmierca. 1998. Etnoarqueología de pastores surandinos. GZC, Buenos Aires. 